\title{
Analytical modelling of metallic circular plates subjected to impulsive loads
}

\author{
R. Zaera, A. Arias, C. Navarro* \\ Department of Mechanical Engineering, Carlos III University of Madrid, Avda. de la Universidad 30, 28911 Leganés, Madrid, Spain
}

\begin{abstract}
This paper presents an analytical approach to the dynamic response of metallic circular plates subjected to impulsive loads. It is based on the plate energy balance equation and assumes that the plate material behaves viscoplastically. The proposed method permits a consideration of the influence of the different terms of the kinetic energy and the plastic work of the plate. A yield criterion is proposed, which involves the coupled effect of the radial and circumferential internal force resultants. By applying the normality rule, the distribution of the bending moments and membrane forces inside the plate are computed. For model validation, its analytical predictions are compared with experimental results.
\end{abstract}

Keywords: Energy methods; Impact; Impulsive loading; Plate; Viscoplastic

\section{Introduction}

In various engineering applications, mechanical elements may be subjected to impacts of foreign objects or to pressure pulses caused by explosions. These involve inertial effects, finite deformations and non-linear behaviour of the materials. So far, the available analytical models concern solids of simple geometric shape, boundary conditions and applied loads, and simplifying hypotheses of the problem are commonly used to allow its analytical treatment. One of the structural problems widely analysed in the past has been that of a metallic circular plate fully clamped around its outer boundary and subjected to transverse impulsive loads. This problem has been stated in different ways, the most usual being to integrate over the plate the momentum equations obtained from a differential plate element (Guowei et al., 1999; Jones, 1989; Quanlin, 1988; Jones, 1968) or to establish energy balance equations (Perrone and Badhra, 1984; Hui and Oliveira, 1986; Woodward et al., 1989). Other workers (Shen and Jones, 1993; den Reijer, 1991) proposed plate deformation patterns using fixed or mobile plastic hinges, applying the momentum equations to the finite plate portions separated by hinges, and movement compatibility conditions.

\footnotetext{
* Corresponding author. Tel.: +34 9162494 91; fax: +34 916249430.

Email address: navarro@ing.uc3m.es (C. Navarro).
} 


\begin{tabular}{|c|c|}
\hline \multicolumn{2}{|c|}{ Nomenclature } \\
\hline $\mathrm{d} s$ & mid-plane differential length in the radial direction \\
\hline$E_{\mathrm{c}}$ & kinetic energy \\
\hline$E_{\mathrm{c}}^{\mathrm{t}}$ & translation kinetic energy \\
\hline$E_{\mathrm{c}}^{\mathrm{r}}$ & rotation kinetic energy \\
\hline$H$ & plate thickness \\
\hline$I$ & impulse \\
\hline$I$ & dimensionless impulse \\
\hline$M_{\mathrm{p}}$ & fully plastic bending moment per unit length \\
\hline$M_{\mathrm{r}}$ & radial bending moment per unit length \\
\hline$M_{\theta}$ & circumferential bending moment per unit length \\
\hline$N_{\mathrm{p}}$ & fully plastic membrane force per unit length \\
\hline$N_{\mathrm{r}}$ & radial membrane force per unit length \\
\hline$p$ & pressure \\
\hline$r$ & radial coordinate \\
\hline$R$ & plate radius \\
\hline$t$ & time \\
\hline$t_{\text {final }}$ & total time of the deformation process \\
\hline$u$ & transversal displacement of the mid-plane \\
\hline$u_{\mathrm{o}}$ & axis transversal displacement \\
\hline$u_{\mathrm{o}}^{\max }$ & final permanent transverse displacement \\
\hline$W_{\mathrm{e}}$ & external work \\
\hline$W_{\mathrm{p}}$ & plastic work \\
\hline$W_{\mathrm{p}}^{\varepsilon_{\mathrm{r}}}$ & membrane force plastic work \\
\hline$W_{\mathrm{p}}^{\mathrm{p}}$ & bending plastic work \\
\hline$W_{\mathrm{p}}^{\chi_{\mathrm{r}}}$ & radial bending plastic work \\
\hline$W_{\mathrm{p}}^{\mathrm{p}} \mathrm{z}_{\theta}$ & circumferential bending plastic work \\
\hline & differential angle in radial direction \\
\hline & differential angle in circumferential direction \\
\hline$\varepsilon_{\mathrm{r}}$ & mid-plane radial strain \\
\hline$\varepsilon$ & equivalent plastic strain \\
\hline$\phi$ & shape function \\
\hline$\rho$ & plate density \\
\hline$\varrho_{\mathrm{r}}$ & radial curvature radius \\
\hline & circumferential curvature radius \\
\hline & elastic limit \\
\hline$\sigma$ & equivalent stress \\
\hline$\theta$ & circumferential coordinate \\
\hline$\Theta$ & gyration angle on the meridian plane \\
\hline & radial curvature \\
\hline$\chi_{\theta}$ & circumferential curvature \\
\hline$\sigma_{\mathrm{o}}, \dot{\varepsilon}$ & $n$ parameters of the Cowper-Symonds equation \\
\hline
\end{tabular}

Given the complexity of the problem, it is commonly assumed that certain terms of the stated equations are not relevant, and so plate rotatory inertia is one of the most controversial terms: some authors neglect it 
(Jones, 1968; Perrone and Badhra, 1984; Hui and Oliveira, 1986) while others take full account of this effect (Shen and Jones, 1993; den Reijer, 1991). The influence of the membrane forces is another matter of discrepancy: Jones (1968), Hui and Oliveira (1986) and Shen and Jones (1993) include this force whereas Guowei et al. (1999) do not. No fixed criteria have been adopted for the incorporation of all these effects in the models.

The analytical approach to the problem also requires a knowledge of the value of the internal force resultants in each plate section. den Reijer (1991) considers that these resultants have constant values in the plate. Woodward et al. (1989) determine the value of some of these resultants and then assume the others as a fraction of them. More sophisticated models (Hodge, 1960; Shen and Jones, 1993) give different yielding criteria depending on whether radial or circumferential bending moments are considered. Yet another model (Guowei et al., 1999) considers a yield criterion involving exclusively bending moments.

The model presented here includes each of the following effects: translation and rotatory inertias, membrane force, and the radial and circumferential bending moments. A yield criterion involving radial membrane force, radial bending moment and circumferential bending moment is also proposed, the normality rule permitting a calculation of these forces during the process of deformation.

\section{Analytical model}

Consider a metallic circular plate with uniform conditions around its outer boundary and subjected to impulsive transverse forces acting on one of its faces (see, for instance, Fig. 1). The basic energy balance equation of the problem may be written as

$$
\dot{W}_{\mathrm{e}}=\dot{E}_{\mathrm{c}}+\dot{W}_{\mathrm{p}}
$$

where $\dot{W}_{\mathrm{e}}, \dot{E}_{\mathrm{c}}$ and $\dot{W}_{\mathrm{p}}$ are respectively the rates of the external load work, kinetic energy, and energy dissipation by plastic deformation. All these terms may be obtained from the displacement field at mid-plane points of the plate. This field can be described in modal form as the product of a function of time $u_{\mathrm{o}}$, representing the transverse displacement of the plate centre, and a shape function $\phi$ of the radial distance $r$ of the considered point. The assumed displacement field should satisfy the plate boundary conditions, so the displacement at any point on the mid-plane of the plate is given by

$$
u=u_{\mathrm{o}}(t) \phi(r)
$$

Only transverse displacement at the mid-plane points are considered, following Griffith and Vanzant (1961), who observed that radial displacements become negligible in comparison with transverse ones in this type of problem.

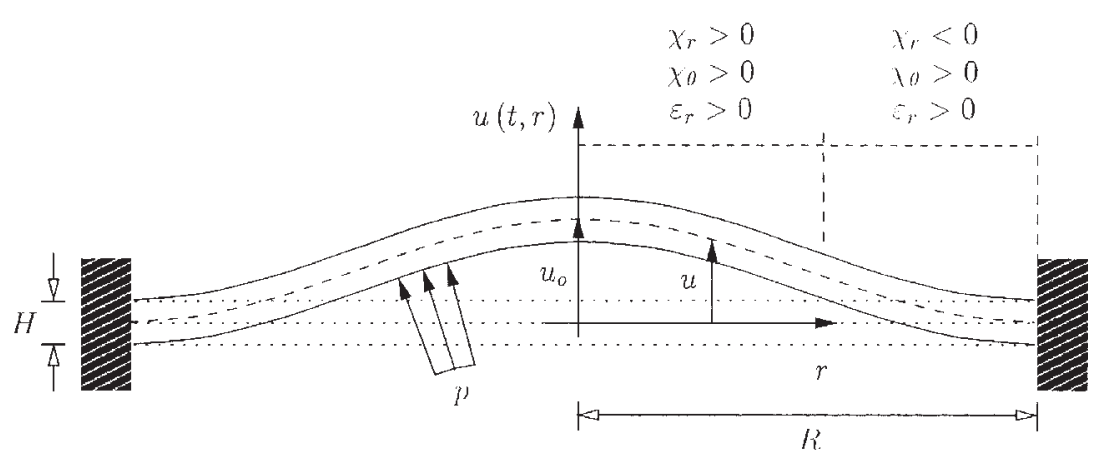

Fig. 1. Meridian section and displacement field of the plate. 


\subsection{External load work rate}

Assuming that boundary reactions produce no mechanical work and that only external pressure is applied on one of the plate faces, i.e., to model the effect of an explosion or a pressure pulse during operation conditions, the work rate of the external forces becomes

$$
\dot{W}_{\mathrm{e}}=\int_{0}^{R} \int_{0}^{2 \pi} p \dot{u}_{\mathrm{o}} \phi r \mathrm{~d} r \mathrm{~d} \theta
$$

where $p$ is the external pressure, $R$ the radius of the plate and $\theta$ the circumferential coordinate. If the pressure is assumed to be uniform, Eq. (3) reduces to

$$
\dot{W}_{\mathrm{e}}=2 \pi p \int_{0}^{R} \dot{u}_{\mathrm{o}} \phi r \mathrm{~d} r
$$

\subsection{Kinetic energy rate}

To evaluate this term, both of its components (translation and rotatory energies) are calculated separately. The elemental plate translation kinetic energy is given by

$$
\mathrm{d} E_{\mathrm{c}}^{\mathrm{t}}=\frac{1}{2} \mathrm{~d} m\left(\frac{\mathrm{d} u}{\mathrm{~d} t}\right)^{2}=\frac{1}{2} \rho H r \mathrm{~d} r \mathrm{~d} \theta\left(\dot{u}_{\mathrm{o}} \phi\right)^{2}
$$

where $H$ is the plate thickness and $\rho$ the material density.

The elemental plate rotatory kinetic energy may be written as

$$
\mathrm{d} E_{\mathrm{c}}^{\mathrm{r}}=\frac{1}{2} \mathrm{~d} I_{\theta} \dot{\Theta}^{2}=\frac{1}{2} \frac{\rho H^{3}}{12} r \mathrm{~d} r \mathrm{~d} \theta\left(\frac{\dot{u}_{\mathrm{o}} \phi^{\prime}}{1+u_{\mathrm{o}}^{2} \phi^{\prime 2}}\right)^{2}
$$

where $\mathrm{d} I_{\theta}$ is the moment of inertia of the considered differential element about a circumferential axis passing through its mass centre, $\phi^{\prime}$, the shape function derivative with respect to the radial coordinate $r$ and $\dot{\Theta}$ is the angular speed about that axis which, in turn, may be calculated by differentiating with respect to time in

$$
\Theta=\arctan \left(\frac{\partial u}{\partial r}\right)=\arctan \left(u_{\mathrm{o}} \phi^{\prime}\right)
$$

Thus the plate translation and rotatory kinetic energies can now be directly obtained by integrating $\mathrm{d} E_{\mathrm{c}}^{\mathrm{t}}$ and $\mathrm{d} E_{\mathrm{c}}^{\mathrm{r}}$ over the whole plate. Then differentiating both energies with respect to time, the kinetic rate term of Eq. (1) finally gives

$$
\dot{E}_{\mathrm{c}}=\dot{E}_{\mathrm{c}}^{\mathrm{t}}+\dot{E}_{\mathrm{c}}^{\mathrm{r}}
$$

where

$$
\dot{E}_{\mathrm{c}}^{\mathrm{t}}=2 \pi \rho H \int_{0}^{R} \dot{u}_{\mathrm{o}} \ddot{u}_{\mathrm{o}} \phi^{2} r \mathrm{~d} r
$$

and

$$
\dot{E}_{\mathrm{c}}^{\mathrm{r}}=\rho \pi H^{3} \int_{0}^{R}\left[\frac{1}{6} \dot{u}_{\mathrm{o}} \ddot{u}_{\mathrm{o}} \frac{\phi^{\prime 2}}{\left(1+u_{\mathrm{o}}^{2} \phi^{\prime 2}\right)^{2}}-\frac{1}{3} u_{\mathrm{o}} \dot{u}_{\mathrm{o}}^{3} \frac{\phi^{\prime 4}}{\left(1+u_{\mathrm{o}}^{2} \phi^{\prime 2}\right)^{3}}\right] r \mathrm{~d} r
$$




\subsection{Plastic work rate}

Plate plastic work rate may be calculated from the following forces per unit length (Fig. 2): the radial bending moment $M_{\mathrm{r}}$, the circumferential bending moment $M_{\theta}$ and the radial membrane force $N_{\mathrm{r}}$. Plastic work rate due to shear force can be neglected in plates with $R / H>2$ (Jones, 1989), and the circumferential membrane force may be neglected if radial displacements are small (Griffith and Vanzant, 1961). Geometric parameters associated with those two bending moments and membrane force are: the radial curvature $\chi_{\mathrm{r}}$, the circumferential curvature $\chi_{\theta}$ and the longitudinal radial strain at mid-plane points $\varepsilon_{\mathrm{r}}$. Curvature values may be deduced from the deformed plate geometry as

$$
\begin{aligned}
& \chi_{\mathrm{r}}=-\frac{u_{\mathrm{o}} \phi^{\prime \prime}}{\left(1+u_{\mathrm{o}}^{2} \phi^{\prime 2}\right)^{3 / 2}} \\
& \chi_{\theta}=-\frac{u_{\mathrm{o}} \phi^{\prime}}{r\left(1+u_{\mathrm{o}}^{2} \phi^{\prime 2}\right)^{1 / 2}}
\end{aligned}
$$

At point $r=0$, the circumferential curvature is

$$
\left.\chi_{\theta}\right|_{r=0}=-u_{0} \phi^{\prime \prime}
$$

The Cauchy strain at mid-plane points $\varepsilon_{\mathrm{r}}$ may be written as

$$
\varepsilon_{\mathrm{r}}=\frac{\mathrm{d} s-\mathrm{d} r}{\mathrm{~d} r}=\left(1+u_{\mathrm{o}}^{2} \phi^{\prime 2}\right)^{1 / 2}-1
$$

where $\mathrm{d} s$ is the differential element length measured in the radial direction on the mid-plane. The elemental plastic work rate due to radial membrane force $\mathrm{d} \dot{W}_{\mathrm{p}}^{\varepsilon_{\mathrm{r}}}$ becomes

$$
\mathrm{d} \dot{W}_{\mathrm{p}}^{\varepsilon_{\mathrm{r}}}=N_{\mathrm{r}} \dot{\varepsilon}_{\mathrm{r}} r \mathrm{~d} r \mathrm{~d} \theta
$$

so the plate plastic work rate for this force may be computed by integration of Eq. (15)

$$
\dot{W}_{\mathrm{p}}^{\varepsilon_{\mathrm{r}}}=2 \pi \int_{0}^{R} u_{\mathrm{o}} \dot{u}_{\mathrm{o}} N_{\mathrm{r}} \frac{\phi^{\prime 2}}{\left(1+u_{\mathrm{o}}^{2} \phi^{\prime 2}\right)^{1 / 2}} r \mathrm{~d} r
$$

The elemental plastic work rate corresponding to radial curvature $\dot{W}_{\mathrm{p}}^{\chi_{\mathrm{r}}}$ is

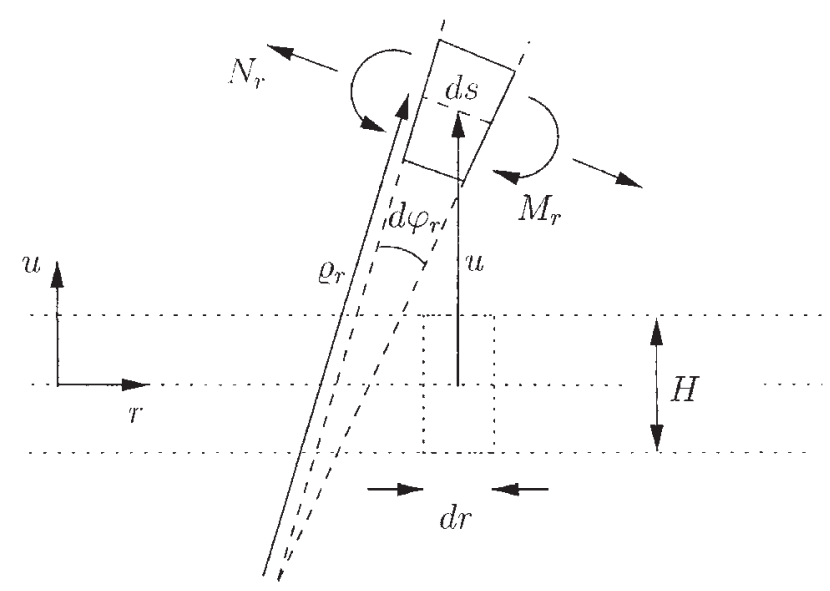

Fig. 2. Deformation of a differential element of the plate on the meridian plane. 


$$
\mathrm{d} \dot{W}_{\mathrm{p}}^{\chi_{\mathrm{r}}}=M_{\mathrm{r}} \frac{\mathrm{d}}{\mathrm{d} t}\left(\mathrm{~d} \varphi_{\mathrm{r}}\right) r \mathrm{~d} \theta=M_{\mathrm{r}} \frac{\mathrm{d}}{\mathrm{d} t}\left(\frac{\mathrm{d} s}{\varrho_{\mathrm{r}}}\right) r \mathrm{~d} \theta=M_{\mathrm{r}}\left(\chi_{\mathrm{r}} \dot{\mathrm{d}} s+\dot{\chi}_{\mathrm{r}} \mathrm{d} s\right) r \mathrm{~d} \theta
$$

$\mathrm{d} \varphi_{\mathrm{r}}$ being the angle between two sections of the element and $\varrho_{\mathrm{r}}$ the curvature radius on the meridian plane (see Fig. 2). Integrating over the whole plate yields

$$
\dot{W}_{\mathrm{p}}^{\chi_{\mathrm{r}}}=2 \pi \int_{0}^{R} M_{\mathrm{r}}\left(\chi_{\mathrm{r}} \dot{\mathrm{d}} s+\dot{\chi}_{\mathrm{r}} \mathrm{d} s\right) r
$$

where

$$
\begin{aligned}
& \mathrm{d} s=\left(1+u_{\mathrm{o}}^{2} \phi^{\prime 2}\right)^{1 / 2} \mathrm{~d} r \\
& \dot{\mathrm{d}} s=u_{\mathrm{o}} \dot{u}_{\mathrm{o}} \frac{\phi^{\prime 2}}{\left(1+u_{\mathrm{o}}^{2} \phi^{\prime 2}\right)^{1 / 2}} \mathrm{~d} r \\
& \dot{\chi}_{\mathrm{r}}=\frac{3 u_{\mathrm{o}}^{2} \dot{u}_{\mathrm{o}} \phi^{\prime 2} \phi^{\prime \prime}}{\left(1+u_{\mathrm{o}}^{2} \phi^{\prime 2}\right)^{5 / 2}}-\frac{\dot{u}_{\mathrm{o}} \phi^{\prime \prime}}{\left(1+u_{\mathrm{o}}^{2} \phi^{\prime 2}\right)^{3 / 2}}
\end{aligned}
$$

As before, the elemental plastic work rate due to the circumferential curvature $\mathrm{d} \dot{W}_{\mathrm{p}}^{\chi_{\theta}}$ is given by

$$
\mathrm{d} \dot{W}_{\mathrm{p}}^{\chi_{\theta}}=M_{\theta} \frac{\mathrm{d}}{\mathrm{d} t}\left(\mathrm{~d} \varphi_{\theta}\right) \mathrm{d} r=M_{\theta} \frac{\mathrm{d}}{\mathrm{d} t}\left(\frac{r \mathrm{~d} \theta}{\varrho_{\theta}}\right) \mathrm{d} r=M_{\mathrm{r}} \dot{\chi}_{\theta} r \mathrm{~d} r \mathrm{~d} \theta
$$

where $\mathrm{d} \varphi_{\theta}$ is the angle between two sections separated $r \mathrm{~d} \theta$ on the second principal curvature plane and $\varrho_{\theta}$ the corresponding curvature radius. Integrating over the plate gives

$$
\dot{W}_{\mathrm{p}}^{\chi_{\theta}}=2 \pi \int_{0}^{R} M_{\theta} \dot{\chi}_{\theta} r \mathrm{~d} r
$$

where

$$
\dot{\chi}_{\theta}=\frac{u_{\mathrm{o}}^{2} \dot{u}_{\mathrm{o}} \phi^{\prime 3}}{r\left(1+u_{\mathrm{o}}^{2} \phi^{\prime 2}\right)^{3 / 2}}-\frac{\dot{u}_{\mathrm{o}} \phi^{\prime}}{r\left(1+u_{\mathrm{o}}^{2} \phi^{\prime 2}\right)^{1 / 2}}
$$

which at $r=0$ takes the value

$$
\left.\dot{\chi}_{\theta}\right|_{r=0}=-\dot{u}_{\mathrm{o}} \phi^{\prime \prime}
$$

Adding the Eqs. (16), (18) and (23), the total plastic work rate $\dot{W}_{\mathrm{p}}$ is finally obtained. So the energy balance relationship (1) can be written finally as a differential equation only of the variable $u_{\mathrm{o}}$

$$
f\left(u_{\mathrm{o}}, \dot{u}_{\mathrm{o}}, \ddot{u}_{\mathrm{o}}\right)=0
$$

This differential equation, as well as the computation of all the above-mentioned integrals can be solved numerically.

\section{Internal forces}

To compute the internal force resultants, the normality rule of the plasticity theory has been used, assuming proportionality between the vector $\left(\dot{\varepsilon}_{\mathrm{r}}, \dot{\chi}_{\mathrm{r}}, \dot{\chi}_{\theta}\right)$ and the vector normal to the yield surface at point $\left(N_{\mathrm{r}}, M_{\mathrm{r}}, M_{\theta}\right)$. In our model a new yield criterion involving the three internal force resultants is proposed 


$$
\left(\frac{N_{\mathrm{r}}}{N_{\mathrm{p}}}\right)^{2}+\left(\frac{M_{\mathrm{r}}}{M_{\mathrm{p}}}\right)^{2}+\left(\frac{M_{\theta}}{M_{\mathrm{p}}}\right)^{2}-\frac{M_{\mathrm{r}} M_{\theta}}{M_{\mathrm{p}}^{2}}=1
$$

where $N_{\mathrm{p}}$ and $M_{\mathrm{p}}$ are respectively the fully plastic membrane force and bending moment

$$
\begin{aligned}
& N_{\mathrm{p}}=\sigma_{y} H \\
& M_{\mathrm{p}}=\sigma_{y} \frac{H^{2}}{4}
\end{aligned}
$$

This criterion has the advantage of being continuous facilitating the implementation of the normality rule. The corresponding yield surface Fig. 3 is convex and verifies the Drucker's stability postulate.

To understand better the physical meaning of the criterion, the intersections of the ellipsoid with the coordinate planes are studied. For instance, if $M_{\theta}$ were zero the yield criterion would reduce to

$$
\left(\frac{N_{\mathrm{r}}}{N_{\mathrm{p}}}\right)^{2}+\left(\frac{M_{\mathrm{r}}}{M_{\mathrm{p}}}\right)^{2}=1
$$

To study the plastic behaviour of beams, Jones (1989) used the simplified stress distribution on a beam section outlined in Fig. 4a, and applying Von Mises criterion, obtained the following relationship between $N_{\mathrm{r}}$ and $M_{\mathrm{r}}$

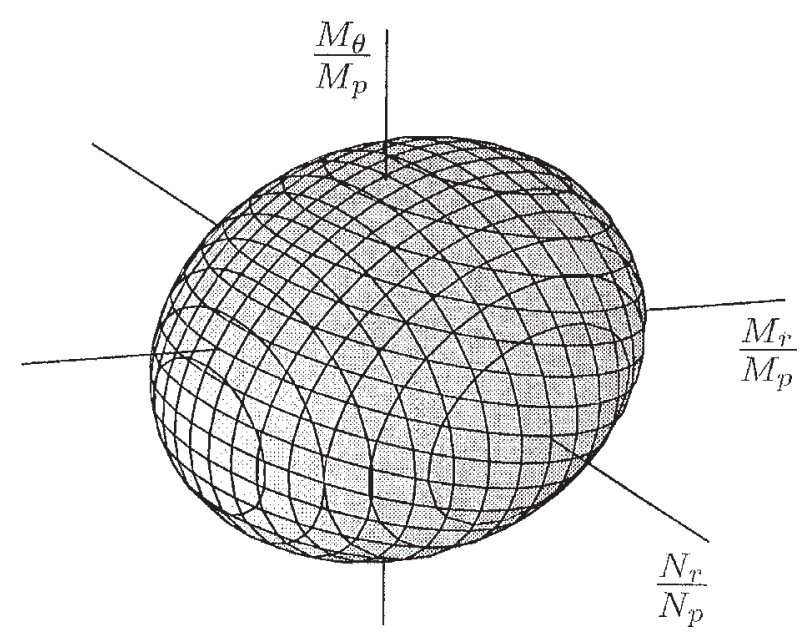

Fig. 3. Yield surface in $N_{\mathrm{r}}, M_{\mathrm{r}}$ and $M_{\theta}$ space.

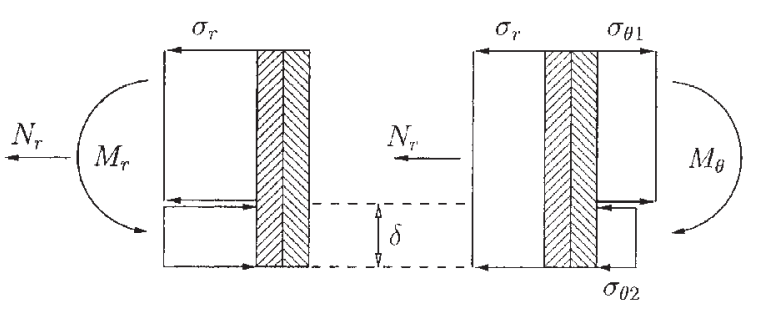

(a)

(b)

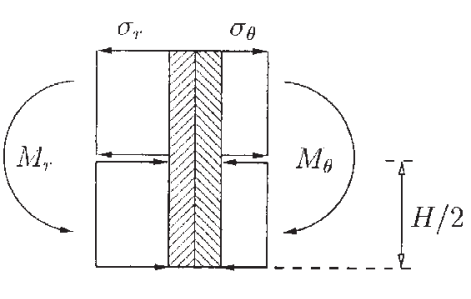

(c)

Fig. 4. Distributions of radial and circumferential stresses for coupled internal force resultants $N_{\mathrm{r}} \quad M_{\mathrm{r}}(a), N_{\mathrm{r}} \quad M_{\theta}(b)$ and $M_{\mathrm{r}} \quad M_{\theta}(c)$. 


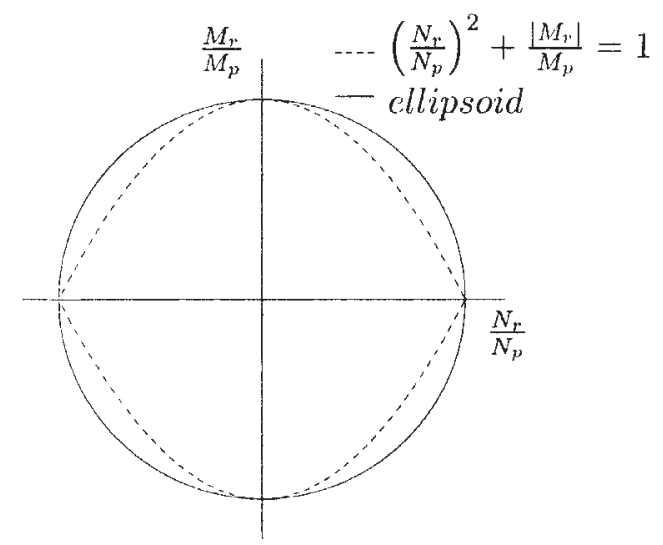

Fig. 5. Yield ellipsoid intersection with $M_{\theta}=0$ plane and Eq. (31).

$$
\left(\frac{N_{\mathrm{r}}}{N_{\mathrm{p}}}\right)^{2}+\frac{\left|M_{\mathrm{r}}\right|}{M_{\mathrm{p}}}=1
$$

A comparison between results deduced from Eqs. (30) and (31) is shown in Fig. 5. The maximum radial difference between the two curves is less than $13 \%$.

For other combinations of any other two internal force resultants, we propose to use the stress distributions shown in Fig. $4 \mathrm{~b}$ and c. If Von Mises criterion were used again, the following relationships would be obtained

$$
\begin{aligned}
& \left(\frac{N_{\mathrm{r}}}{N_{\mathrm{p}}}\right)^{2}+\frac{\left|M_{\theta}\right|}{M_{\mathrm{p}}}\left(1-\frac{3 N_{\mathrm{r}}^{2}}{4 N_{\mathrm{p}}^{2}}\right)^{1 / 2}=1 \\
& \left(\frac{M_{\mathrm{r}}}{M_{\mathrm{p}}}\right)^{2}+\left(\frac{M_{\theta}}{M_{\mathrm{p}}}\right)^{2}-\frac{M_{\mathrm{r}} M_{\theta}}{M_{\mathrm{p}}^{2}}=1
\end{aligned}
$$

Figs. 6 and 7 compare the results deduced from Eqs. (32) and (33) and those from Eq. (27). Fig. 6 illustrates how close (radial differences less than 6\%) are the results provided by Eqs. (32) and (27) whereas Fig. 7 shows the total coincidence of results obtained from Eqs. (33) and (27).

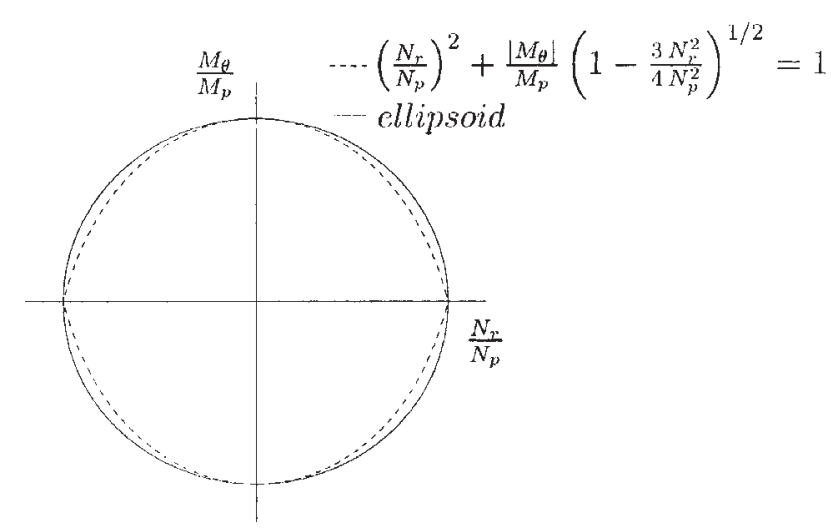

Fig. 6. Yield ellipsoid intersection with $M_{\mathrm{r}}=0$ plane and Eq. (32). 


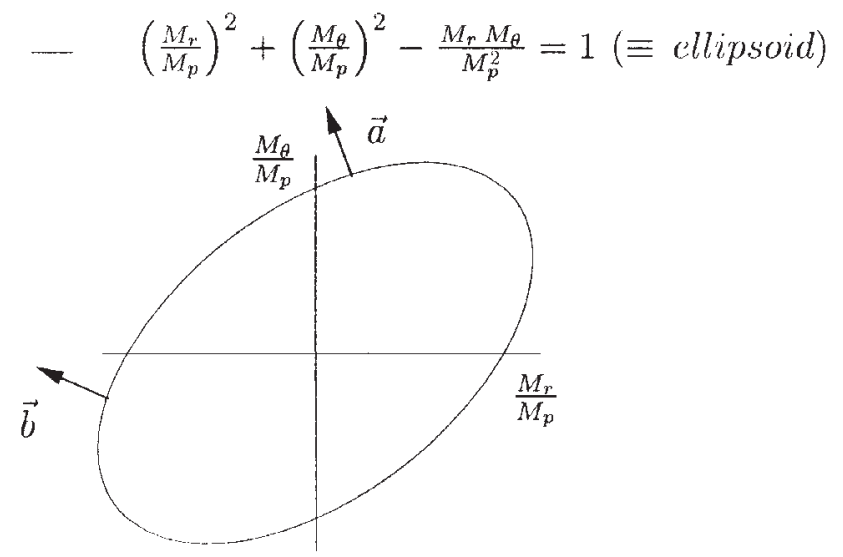

Fig. 7. Yield ellipsoid intersection with $N_{\mathrm{r}}=0$ plane and Eq. (33).

In view of the above, Eq. (27) is chosen as the yield criterion for the model. Applying the normality rule of plasticity, the following values of the internal force resultants are obtained

$$
\begin{aligned}
& N_{\mathrm{r}}=\sqrt{ } 3 \frac{N_{\mathrm{p}}^{2} \dot{\mathrm{\varepsilon}}_{\mathrm{r}}}{\left(3 N_{\mathrm{p}}^{2} \dot{\varepsilon}_{\mathrm{r}}^{2}+4 M_{\mathrm{p}}^{2}\left(\dot{\chi}_{\mathrm{r}}^{2}+\dot{\chi}_{\theta}^{2}+\dot{\chi}_{\mathrm{r}} \dot{\chi}_{\theta}\right)\right)^{1 / 2}} \\
& M_{\mathrm{r}}=\frac{2 \sqrt{ } 3}{3} \frac{M_{\mathrm{p}}^{2}\left(2 \dot{\chi}_{\mathrm{r}}+\dot{\chi}_{\theta}\right)}{\left(3 N_{\mathrm{p}}^{2} \dot{\varepsilon}_{\mathrm{r}}^{2}+4 M_{\mathrm{p}}^{2}\left(\dot{\chi}_{\mathrm{r}}^{2}+\dot{\chi}_{\theta}^{2}+\dot{\chi}_{\mathrm{r}} \dot{\chi}_{\theta}\right)\right)^{1 / 2}} \\
& M_{\theta}=\frac{2 \sqrt{ } 3}{3} \frac{M_{\mathrm{p}}^{2}\left(2 \dot{\chi}_{\theta}+\dot{\chi}_{\mathrm{r}}\right)}{\left(3 N_{\mathrm{p}}^{2} \dot{\varepsilon}_{\mathrm{r}}^{2}+4 M_{\mathrm{p}}^{2}\left(\dot{\chi}_{\mathrm{r}}^{2}+\dot{\chi}_{\theta}^{2}+\dot{\chi}_{\mathrm{r}} \dot{\chi}_{\theta}\right)\right)^{1 / 2}}
\end{aligned}
$$

\section{Model validation}

To validate the model, an example was analysed: a metallic circular plate clamped at its outer border, subjected to explosive charge pressure on one of its faces. Experimental results are available for this problem (Bodner and Symonds, 1979) which have been used to validate analytical models (Perrone and Badhra, 1984; Shen and Jones, 1993). Bodner and Symonds (1979) used two high strain rate sensitive materials for their experiments: hot rolled mild steel (ASTM.A415) and 99.2\% purity titanium (Ti-50A). The geometry parameters of the plates are shown in Table 1, as well as the material properties assuming that both materials behave following the Cowper-Symonds hardening equation

$$
\frac{\sigma}{\sigma_{\mathrm{o}}}=1+\left(\frac{\dot{\varepsilon}}{\dot{\varepsilon}_{\mathrm{o}}}\right)^{1 / n}
$$

$\sigma$ and $\dot{\varepsilon}$ being the effective stress and the effective plastic strain rate, respectively. The yield stress that appears in Eqs. (28) and (29) was obtained from Eq. (37), assuming $\dot{\varepsilon}_{\mathrm{r}}$ as the effective plastic strain rate. The shape function $\phi(r)$ used in the model was that proposed by Westine and Baker (1974) for fully clamped circular plates 
Table 1

Properties of the materials used by Bodner and Symonds (1979)

\begin{tabular}{lll}
\hline Dimension, property & ASTM.A415 steel & Ti A titanium \\
\hline$R(\mathrm{~m})$ & 0.0318 & 0.0318 \\
$H(\mathrm{~m})$ & 0.00193 & 0.00234 \\
$\rho\left(\mathrm{kg} / \mathrm{m}^{3}\right)$ & 7850 & 4520 \\
$\sigma_{\mathrm{o}}(\mathrm{MPa})$ & 223 & 251 \\
$\dot{\varepsilon}_{\mathrm{o}}\left(\mathrm{s}^{-1}\right)$ & 40 & 120 \\
$n$ & 5 & 9 \\
\hline
\end{tabular}

$$
\phi(r)=\frac{1}{2}\left(1+\cos \frac{\pi r}{R}\right)
$$

The pressure pulse caused by the explosive charge was assumed to be triangular, as proposed by Biggs (1964)

$$
p(t)=p_{\max }\left(1-\frac{t}{t_{\text {load }}}\right)
$$

where $t_{\text {load }}$ is the duration of the pulse and $p_{\max }$ the pressure at its beginning. The value of $t_{\text {load }}$ was $10 \mu$ in accordance with the duration of the pulse given by Bodner and Symonds (1979). These authors also measured the final permanent displacement at the plate centre as a function of the applied impulse

$$
I=\int p \mathrm{~d} t
$$

Knowing the duration of the event and the acting impulse, $p_{\max }$ can be obtained.

Figs. 8 and 9 show comparisons of the results from the model presented, the experimental ones, and those deduced from the analytical models of Shen and Jones (1993) and Jones (1989), the latter incorporating the viscoplastic behaviour of the material according to the work of Perrone and Badhra (1984). Both

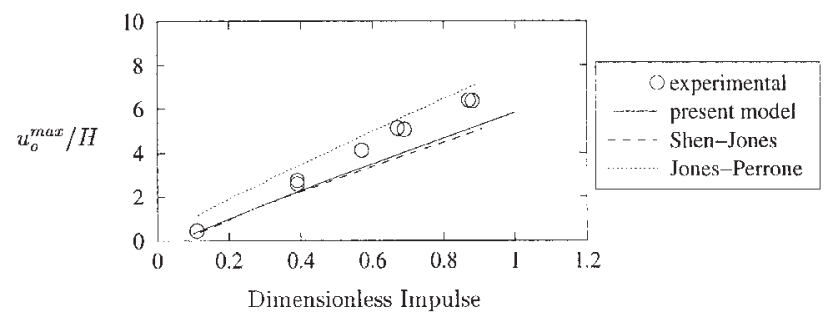

Fig. 8. Comparison of model prediction with other models and experimental results for a steel plate.

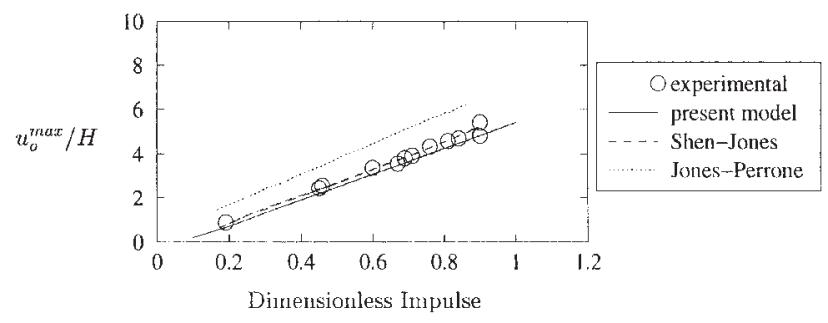

Fig. 9. Comparison of model prediction with other models and experimental results for a titanium plate. 
figures show dimensionless final permanent displacement at the centre of the plate $u_{\mathrm{o}}^{\max }$ as a function of the dimensionless impulse given by

$$
I=\frac{I}{\sqrt{ } \rho \sigma_{\mathrm{o}} H^{2}}
$$

Note that the results of the proposed model are very close to those deduced from Shen and Jones model.

\section{Results and discussion}

One of the main advantages of the proposed model is that it allows a separate study of the influence of each component of the inertial and plastic work rates on the dynamic response of the plate. Different studies of ASTM.A415 steel plate subjected to impulsive pressure were carried out. Fig. 10 shows the time evolution of the dimensionless plastic work done by the membrane force and bending moments, as well as the dimensionless axis displacement for a dimensionless impulse $I=0.7$. Membrane force work is of the same order of magnitude as the bending moment work when the plate axis displacement is greater than the thickness, after which the bending plastic work is reduced to only a small fraction of the total plastic work of the plate.

Fig. 11 shows, for a given value of $R$ and different values of $R / H$, the ratio of the membrane force and the total bending moment plastic work. For a particular value of the final plate centre displacement, bending plastic work becomes more important as the plate thickness increases, since the fully plastic bending moment is proportional to the square of the plate thickness (Eq. (29)), while the plastic membrane force depends linearly on this parameter (Eq. (28)). If instead of using $u_{\mathrm{o}}^{\max }$ as the variable, the dimensionless displacement were considered (Fig. 12), all the curves shown in Fig. 11 become very close. This

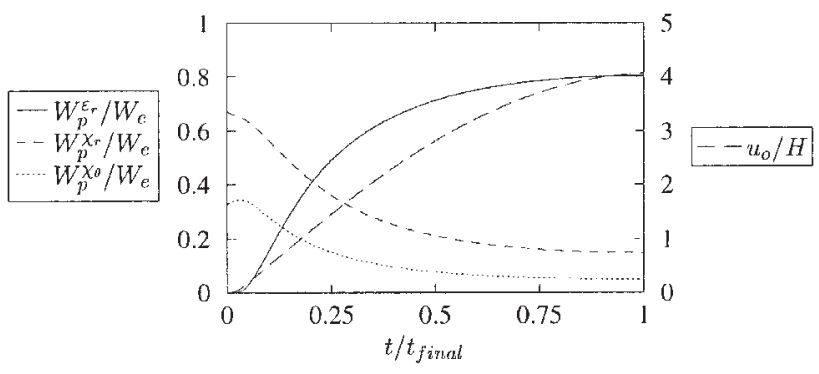

Fig. 10. Time dependence of the dimensionless plastic work done by the three internal force resultants and of the axis displacement (ASTM.A415 steel, $R / H=12, I=0.7$ ).

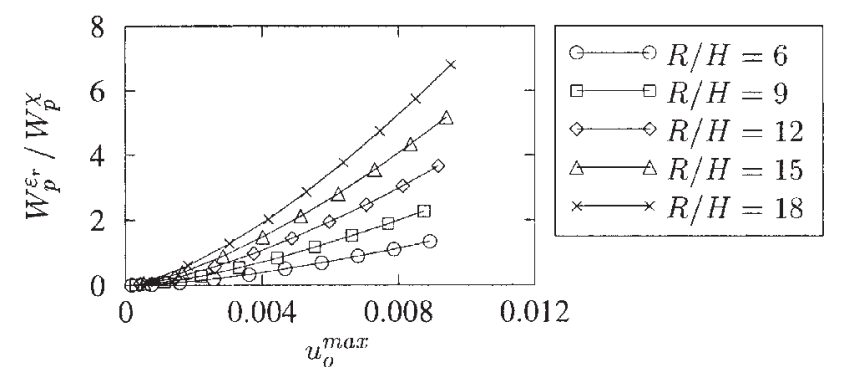

Fig. 11. Dependence of $W_{\mathrm{p}}^{\varepsilon_{\mathrm{r}}} / W_{\mathrm{p}}^{\chi}$ ratio on final permanent transverse displacement for different $R / H$ ratios (ASTM.A415 steel, $R=30$ $\mathrm{mm}, 0<I<1)$. 


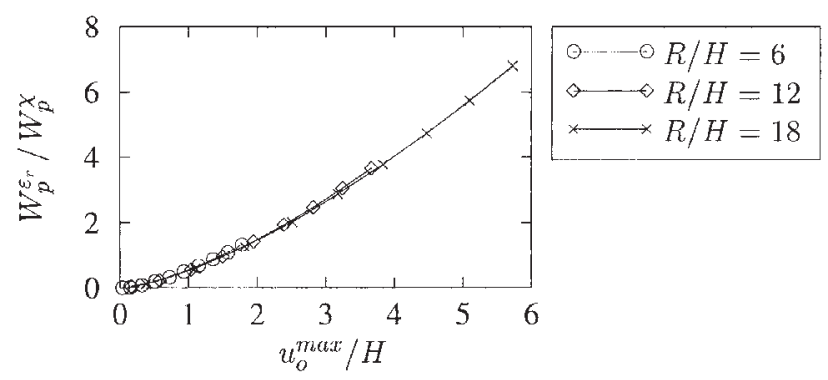

Fig. 12. Dependence of $W_{\mathrm{p}}^{\varepsilon_{\mathrm{r}}} / W_{\mathrm{p}}^{\chi}$ ratio on dimensionless final permanent transverse displacement for different $R / H$ ratios (ASTM.A415 steel, $R=30 \mathrm{~mm}, 0<I<1)$.

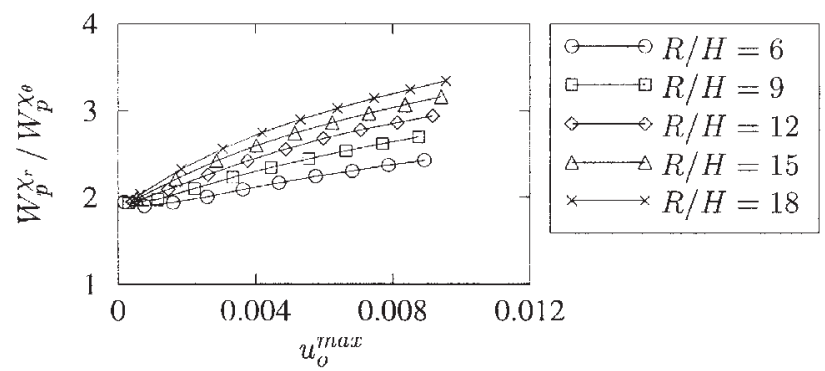

Fig. 13. Dependence of $W_{\mathrm{p}}^{\chi_{\mathrm{r}}} / W_{\mathrm{p}}^{\chi_{\theta}}$ ratio on final permanent transverse displacement for different $R / H$ ratios (ASTM.A415 steel, $R=30$ $\mathrm{mm}, 0<I<1)$.

figure also shows that membrane force plastic work becomes much more important than the bending force for $u_{\mathrm{o}}^{\max }$ greater than $H$.

With regard to the circumferential bending plastic work, Woodward et al. (1989) suggested that its value is equal to that of the radial moment. Fig. 10 shows that although their works are of the same order of magnitude, that of $M_{\mathrm{r}}$ is greater than that of $M_{\theta}$. Fig. 13 also illustrates this tendency, which becomes much clearer as the final displacement and the ratio $R / H$ are greater.

Fig. 14 shows the diagrams of the normalized membrane force and bending moments at four different stages of the event. At the earlier stages, bending moments are more important than the membrane force, especially near the plate axis and clamping plate perimeter where the plate curvature is greater. Around $r=R / 2$, where the slope of the plate is greater (therefore $\dot{\varepsilon}_{\mathrm{r}}$ ), the membrane force reaches its maximum, increasing as the plate deformation increases, rapidly reaching its fully plastic value over almost the whole plate. However, bending moments decrease sharply.

Fig. 14 also shows that there are plate regions where the sign of bending moments and corresponding curvatures are different. For instance, at earlier stages of the event and at points in which the $r$ coordinate is slightly greater than $R / 2$ (plate inflexion point), the radial bending moment is still positive although the radial curvature is negative. This can be explained from vector $\vec{a}$ of Fig. 7. In this zone of the yield surface the circumferential curvature reaches its maximum (bigger slope of the plate) and the radial curvature rate is small (proximity to plate inflexion point) and negative, whereas $M_{\mathrm{r}}$ is positive. The same occurs with the circumferential bending moment near clamping, where negative values of this moment appear, even though the associated curvature is positive (see $\vec{b}$ of Fig. 7).

Regarding the influence of the rotatory kinetic energy on the plate response, its value was $1 \%$ below the translation kinetic energy (Fig. 15), except at the end of the deformation process, where the slope of the shape function is greater and the translation term converges to zero more quickly than the rotatory one. So the effect of the rotatory inertia seems to have little importance in the analysed cases. 

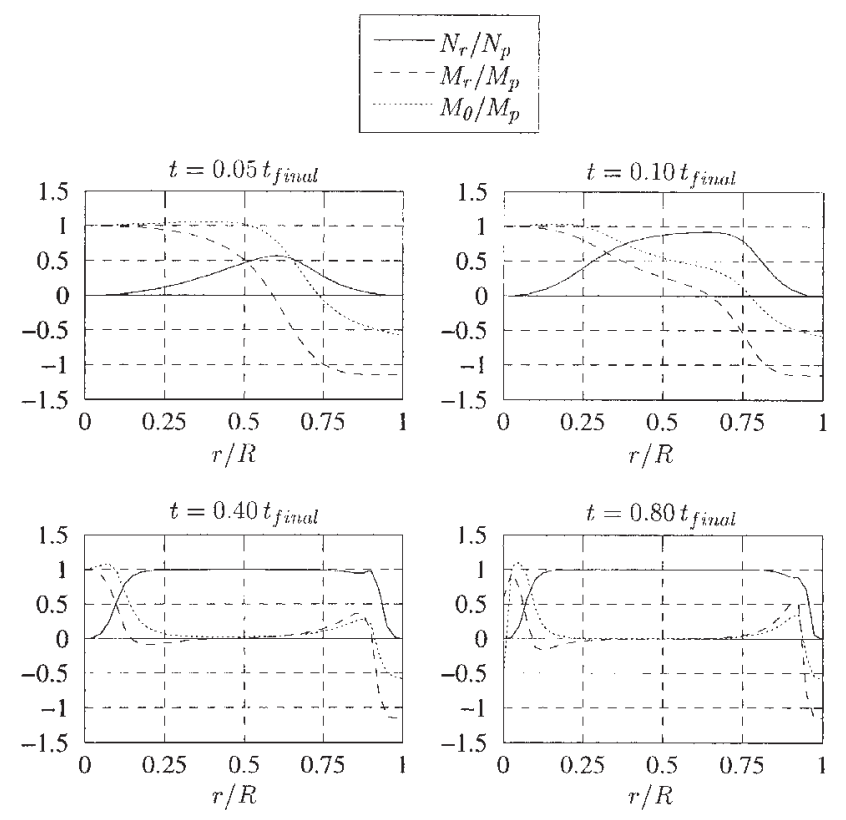

Fig. 14. Diagrams of dimensionless internal force resultants at for instants of the impact process (ASTM.A415 Steel, $R / H=12$, $I=0.7)$.

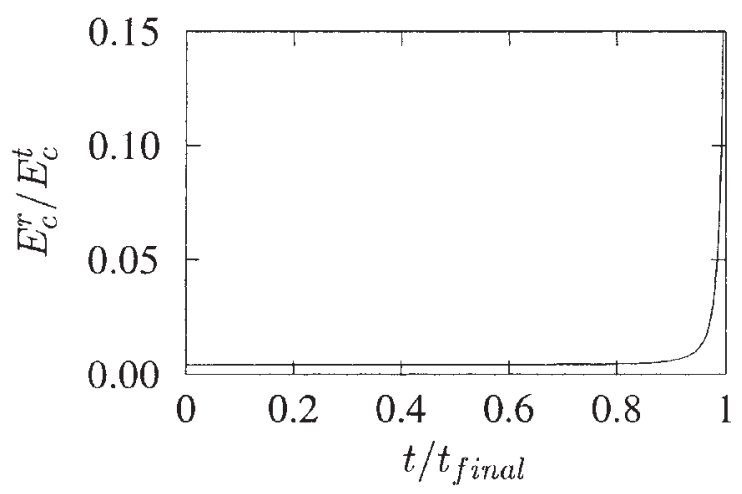

Fig. 15. Time dependence of $E_{\mathrm{c}}^{\mathrm{r}} / E_{\mathrm{c}}^{\mathrm{t}}$ ratio (ASTM.A415 Steel, $R / H=12, I=0.7$ ).

\section{Concluding remarks}

The simplified analytical model of the problem of a metallic circular plate subjected to impulsive loads gives results close to those obtained by experiment. It is based on an energy balance equation, and the main effects (translation and rotatory inertia, the membrane force, and the radial and circumferential bending moments) are included. This allows a separate study of their influence on the dynamic plate response. A yield criterion is also proposed that considers radial membrane force, radial and circumferential bending moments.

The model is used to simulate the effect of external pressures of different values (dimensionless impulse $I$ ranging from 0 to 1 ) on metallic plates (steel or titanium) with different geometries ( $R / H$ from 6 to 18 ), and from the results some conclusions can be drawn. The membrane force should be included in the analysis if the expected final permanent transverse deflection is greater than the plate thickness. For smaller values of 
this parameter, the bending curvature seems to be much more important than the membrane strain at plate mid-plane points. Although the circumferential bending plastic work is less than that of radial bending, it should be incorporated into the model, especially for thick plates and large deflections. Rotatory inertia can be neglected over the range of variables investigated, since its value is much smaller than that of translation.

This analytical model could be extended easily to other axisymetric plates, boundary conditions and loading cases, so annular plates could be analysed with this model, simply by varying the shape function.

\section{Acknowledgements}

The authors are indebted to the Comunidad Autónoma de Madrid for the financial support of this work (project 07N/0004/1998).

\section{References}

Biggs, J.M., 1964. Structural Dynamics. McGraw Hill, New York.

Bodner, S.R., Symonds, S., 1979. Experiments on viscoplastic response of circular plates to impulsive loading. J. Mech. Phys. Solids 27, 91113.

den Reijer, P.C., 1991. Impact on ceramic faced armours. Ph.D. Thesis, Technische Universiteit Delft.

Griffith, J., Vanzant, H., 1961. Large deformation of circular membranes under static and dynamic loads. First International Congress on Experimental Mechanics, New York.

Guowei, M., Iwasaki, S., Miyamoto, Y., Deto, H., 1999. Dynamic plastic behaviour of circular plates using unified yield criterion. Int. J. Solids Struct. 36, 32573275.

Hodge, P.G., 1960. Yield conditions for rotationally symmetric shells under axisymetric loading. J. Appl. Mech. $27,323331$.

Hui, D., Oliveira, J.G., 1986. Dynamic plastic analysis of impulsively loaded viscoplastic rectangular plates with finite deflections. J. Appl. Mech. 53, 667674.

Jones, N., 1968. Impulsive loading of a simple supported circular rigid plastic plate. J. Appl. Mech. 35, 5965.

Jones, N., 1989. Structural Impact. Cambridge University Press, Cambridge.

Perrone, N., Badhra, P., 1984. Simplified large deflection mode solution for impulsively loaded, viscoplastic, circular membrane. J. Appl. Mech. 51, 505509.

Quanlin, J., 1988. Dynamic response of an infinitely large rigid plastic plate impacted by a rigid cylinder with transverse shear and rotatory inertia. Int. J. Impact Engng. 7, 391400.

Shen, W.Q., Jones, N., 1993. Dynamic response and failure of fully clamped circular plates under impulsive loading. Int. J. Impact Engng. 13, 259278.

Westine, P.S., Baker, W.E., 1974. Energy solutions for predicting deformation in blast loaded structures. 19th Explosive Safety Seminar, Florida.

Woodward, R.L., O’Donnell, R.G., Baxter, B.J., Nicol, B., Pattie, S.D., 1989. Energy absorption in the failure of ceramic composite armours. Mater. Forum 13, 174181 